\title{
SENSOR FUSION, GIS AND AI TECHNOLOGIES FOR DISASTER MANAGEMENT
}

\author{
H. $\operatorname{Kemper}^{1 *}$, G. Kemper ${ }^{2}$ \\ ${ }^{1}$ University Bonn / Germany - hannahkemper.speyer@gmail.com \\ ${ }^{2}$ GGS GmbH, Speyer / Germany - kemper@ggs-speyer.de
}

KEY WORDS: Multi-Sensor, Thermal, Disaster Management, GIS, Machine Learning, UAV

\begin{abstract}
:
Modern Disaster Management Systems are based on several columns that combine theory and practice, software, and hardware being under technological advance. In all parts, spatial data is key in order to analyze existing structure, assist in risk assessment and update the information after a disaster incident. This paper focus on technological advances in several fields of spatial analysis putting together the advantages, limitations and technological aspects from well-known or even innovative methods, highlighting the huge potential of nowadays technologies for the field of Disaster Risk Management (DRM).

A focus then is lying on GIS and Remote Sensing technologies that are showing the potential of high-quality sensors and image products that are getting easier to access and captured with recent technology. Secondly, several relevant sensors being thermal or laser-based are introduced pointing out the application possibilities, their limits, and potential fusion of them. Emphasis is further driven to Machine Learning techniques adopted from Artificial Intelligence that improve algorithms for auto-detection and represent an important step forwards to an integrated system of spatial data use in the Disaster Management Cycle. The combination of Multi-Sensor Systems, new Platform technologies, and Machine Learning indeed creates a very important benefit for the future.
\end{abstract}

\section{INTRODUCTION}

Disasters, either natural or man-made, happen more frequently and are often more intense due to the expansion and densification of urban structures and acceleration of climate change (O’Brien et al. 2006; van Aalst 2006; Bouwer 2011.). Every year, many people either die or are injured by disasters, besides that growing economic damage is needed to be handled. The risk appears in places of a human activity where nature is active and people have settlements (Altan and Kemper 2016.). Risk can be seen as a combination of a natural hazard, which takes an effect on exposed people that experience earthquakes, volcanism, tsunamis, weather-disasters, etc. meanwhile in other places natural disasters often occur unrecognized in areas apart from the population (Altan and Kemper 2016.). Urban sprawl and population growth result in the urbanization of areas that can be threatened by several hazards. People accessing these areas are forced to handle with their livelihoods i.e. farming and social circumstances e.g. volcanic soils like the Pululahua area in Northern Ecuador, which offers high fertility (Altan and Kemper 2016.).

Disasters can be based on climatic basis e.g. storms, floods, droughts, and others, meanwhile, geological disasters would contain earthquakes, tsunamis, volcanic eruptions, landslides, and avalanches. Further, biological disasters as epidemic or pandemic diseases (e.g. the Covid 19 pandemic) or plant diseases that lead e.g. to forest dying are crucial examples. Manmade hazards which should be taken into account are

\section{* Contributing author}

(civil) wars, economic crises or political conflicts (Altan and Kemper 2016.).
Modern technologies (hardware) in combination with geospatial data in GIS environment open a wide field for assisting in Disaster Risk Management. Taking into consideration the mitigation, preparation, response and recovery phases of the disaster management cycle (Haigh 2017.) abundant application options are thinkable with nowadays technologies. Crisis Preparedness Plans play a key role in the protection of exposed citizens (Altan and Kemper 2008.) meanwhile disciplines of Photogrammetry, Remote Sensing and Spatial Information Science can contribute in many ways to them. Further, the creation of risk awareness is a key to improve general conditions and measures for disaster risk handling. To be prepared means to have thorough knowledge about the geospatial structure, the mechanism of the disaster, and scenarios to model and train disaster management plans. Geospatial data is one of the basic resources to e.g. detect the critical areas in case of a flood, analyze areas that might be safer than others in case of an earthquake, and to find most suitable places for shelters for homeless people. Looking at the response phase a fast data availability is crucial to successful operation on the ground. It is necessary to guide rescue teams through the situation. Data referencing is, therefore, a fundamental basis to immediately feed the data server of crisis management teams (Altan and Kemper 2016.).

Gathering the abundant methods is needed to guarantee sustainable help to people, whereas interdisciplinary cooperation is highly recommended. The importance of GIS and RS for DRM is surely known within research, but also National and International Institutions or Organizations around the globe work in certain ways with geospatial data like UN SPIDER ${ }^{1}$, the National Entities of Canada (Agrawal 2018.) or the National Unity of Disaster Risk Management in Colombia ${ }^{2}$.

\footnotetext{
${ }^{1}$ http://www.un-spider.org/
} 
Linking disaster risk management to broader sustainable development goals is now envisaged with the "Sendai Framework for Disaster Risk Reduction" (United Nations Office for Disaster Risk Reduction 2015.). However, this is only possible with the knowledge transfer generated through research and periodic assessments that are effectively communicated to society and governments. DRM decision-makers should be provided with evidence of economic, operational, and strategic benefits of using scientific knowledge and information, which address prevention, mitigation, and response actions (e.g., Altan et al., 2010, 2013). UNDRR's Sendai Framework for Disaster Risk Reduction targets the enhancement of several important aspects in the Disaster Risk Management, which further draws attention to the importance of this topic. By outlining seven clear targets and four priorities for action the framework sets new standards for research and policymakers in the field of Disaster Management.

Research goes on combining new sensors in new platforms with better software and create an impact on DRM. Looking to international research group results from Pakistan (Hussain, Arsalan, Siddiqi et al. 2005), China (Wang, Wu, Zhu 2019); Korea (Seongsam, Jonyoung, Jinsoo 2016), or Congresses (Intelligent Systems for Crisis Management Gi4DM 2018) show impressive results for their contribution to an improved DRM.

\section{GIS}

Geographic Information System (GIS) opened up new possibilities for data capture, management, analysis and presentation (FAO) working with Geospatial data, which can be described as information bound to certain locations on a coordinate system referring to the Earth ${ }^{3}$.

Generally the field of Disaster Risk Management has recognized the usefulness and potential of geospatial analysis using GIS. The growing importance of the findings given by research shows that broad implementation of geospatial analysis can be given during all phases of the disaster management cycle (Haigh 2017.) showing potential for mitigating risk analysis, preventive identification of emergency areas and responsive emergency location and quick responsive mapping. This can be implemented in Preparedness Processes of Evacuation Planning, the Identification of High-Risk Areas, and the Response to provide accurate information on the exact location of an emergency context, as the potential is considerably high (Bala et al. 2017.). The number of papers published towards the GIS-based contributions to DRM shows the growing interest and importance of this method. Mainly Floods and Cyclones are given big importance in recent literature (among others Kienberger and Steinbruch 2005; Abbas S.H. et al. 2009; Khan 2013; Forslund 2016.). Further, the landslide susceptibility (Chen et al. 2018.) or Critical Infrastructure can be seen as examples for GIS focused research in the field of DRM. Having a look at participative approaches in Geoinformatics papers about Community-based DRM (Maskrey 2011; Canevari-Luzardo et al. 2017.) and the inclusion of People as

\footnotetext{
${ }^{3}$ https://dictionary.cambridge.org/dictionary/english/geospatial
}

Sensors for Data Contribution (Laituri and Kodrich 2008.) appear to give a new perspective for further bottom-up strategies in the field.

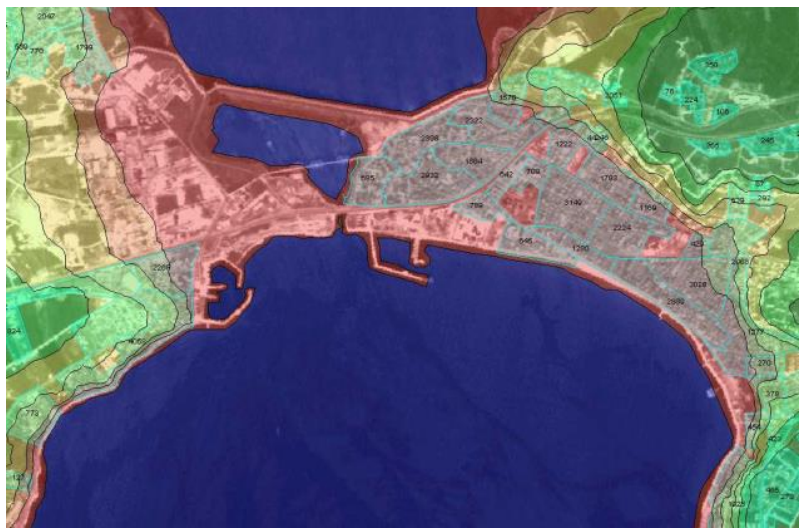

Figure 1: Tsunami Run-up simulation represented as a Map of an area near Istanbul calculated via GIS using land-use data and a DTM (Kemper et.al 2005)

\section{SENSORS}

\subsection{Satellite Images}

Referring to satellite images as gap-filler is not up to date anymore. Satellite imagery offers a reliable and low-cost alternative to cost-intensive aerial images. Further, multi-spectral information (Landsat TM) gets a more detailed view of aspects, which are visibly hidden to us. Meanwhile the spatial resolution becomes better and the demand for free products is rising, geoprocessing and referencing of big data amounts gets better and faster (Altan and Kemper 2016.)

Especially the use of land cover information can give an important extra to Risk Assessments during Preparatory Assessments of an area. They further provide information about the general landscape characteristics of an area and depending on the resolution will be a basis for digitalization works of buildings or roads, which could be realized in a response or mitigation phase (e.g. HOT initiative ${ }^{4}$ )

After a disaster strikes a certain area satellite images are one of the first datasets that are available to the disaster response teams. They contribute crucial impressions from the area and can be taken as a first-glance look on the situation, meanwhile, the use for early-warning is limited (Altan and Kemper 2016.) Several research projects (Xueqiao Huang and John R. Jensen 1997; Alpar et al. 2004; Abdel-Aziz Belal et al. 2014.) working with Satellite imagery proof that the potential of this technology is not reached yet, since new satellites with better spatial and temporal resolution is launched every year (e.g. Sentinel satellites).

\subsection{Radar and Hyperspectral Satellite data}

Beside optical satellite sensors, radar, and hyperspectral sensors deliver data for a specific use. Radar can penetrate clouds

\footnotetext{
${ }^{4}$ https://www.hotosm.org/
} 
and can produce a sufficient terrain model. Typically terrain models generated from Lidar or aerial images have a higher density and better accuracy. Therefore, Radar data is not really used for a basic geo-dataset. However, frequently updated radar surveys are able to deliver minor adjustments using interferometric analyses to highlight small changes in the DTM (Tronin 2010.). These anomalies show stress in the geological layers which indicates geological movements - may be an upcoming earthquake. Radar is a good early warning sensor to indicate geological shock waves. Also after an earthquake, Radar highlights the changes in the terrain, useful information for the rescue teams.

Hyperspectral sensors (Niculescu et al. 2010.) have been developed for monitoring environmental and geological structures. Focused on this specific task, they can support disaster management in all parts. Even the geometric resolution is not high, environmental disasters can be well monitored in order to provide early warning and disaster management.

As also satellite images are, they can be made accessible for the Disaster Management typically a day after capturing. However, the way of data preprocessing is more complex in order to get the first visible result for inspection.

\subsection{Aerial nadir images}

Aerial nadir images have been and are excellent data sources being frequently used in the past for generating spatial data. During the last 60 years, aerial images have been the most dominant source for mapping and geodata management. Today's digital sensors have replaced the analog technology and enable typically RGB and NIR in one flight and quick access for rapid data update. Medium format mapping cameras with 150 MP can be used in UL aircraft, Trikes, and UASs. This widens the range of use for aerial images due to an enhanced range of GSD (0.1-20 cm). Meanwhile for UASs mid format metric cameras up to $150 \mathrm{MP}$ and found their market to generate Orthophotos and aerial maps in highest resolution and accuracy (Altan and Kemper 2016.).

Nadir aerial images show far more details than satellite images do and the date for a flight can be defined according to the needed resolution and the weather conditions. That gives higher flexibility, resolution and accuracy, especially in 3D mapping applications. Big format digital mapping cameras have resolutions up to 450 MPix (Vexcel UltraCam Eagle Mark 3) that can cover wide areas. Midformat cameras meanwhile also cover three times more than old analog ones did; single cameras with $150 \mathrm{MP}$, dual stitched 4-bans solutions Systems with 280 MP or setups for wide corridor mapping with a 3 Camera setup at 35.000 pixels cross-track. Besides that, mid-format mapping cameras have changeable lenses that make them extremely flexible for various needs.

All these camera-setups do an excellent job for preparedness mapping in $3 \mathrm{D}$ as well as monitoring the Disaster in order to assist the rescue teams.

The new mid format cameras that make use CMOS sensors (e.g. Phase One IXM -RS 150) can capture data also under poor and difficult light conditions and are the first choice for

${ }^{5}$ https://www.geospatialworld.net/article/fast-orthophoto-productionusing-the-digital-sensor-system remotely sensed data acquisition in aircraft and UASs. After a mission, the images can be accessed immediately even there is just a core reference. Using direct referencing methods, the image data can be directly be processed and overlayed in a GIS environment to generate rapid information. Inflight processing of images to generate rapid orthophoto on the fly is a technology invented in $2009^{5}$ and since then continuously optimized ${ }^{6}$.

\subsection{Oblique images}

Oblique imaging became very requested within the last years even the way of capturing non-nadir images was already used for many decades. Besides architectural oblique imaging, also for open mining and other special tasks oblique images were used for a long time. For about eight years, there was a higher request for such data, either for homeland-security issues or for internet applications as Bing, Virtual Earth, Google, city planning, and others. With some exceptions, a core georeference to display the data on the approximate place over a map was sufficient. The image quality improved dramatically during the last years and the use of the generated data is not restricted anymore to segments.

Today we can make use of cameras with medium format sensors that capture images with 100-150 MPIX. Not just the higher resolution, also the precise image geometry of such mapping cameras widened the range to use these data also for precise mapping and modeling. The use of the today's captured oblique images is designed for precise 3D City models in order to generate a high dense DSM with an extreme good high accuracy and high redundancy of image data for texturing the facades. For two years, this initiated a strongly growing request for such systems. For the preparedness plan, we can make use of highly accurate oblique cameras system as the OIS-XL4B using 6 PhaseOne cameras each with 100-150 MPix a good way to capture nadir and oblique data in one flight and to extract a high dense city model with all information about the building structures that can assist to generate data for crisis preparedness. In this camera, a nadir 4band system is implemented to get also information as CIR and NDVI (Kemper 2018.).

As shown on the figure 2 there is definitely a certain need for such information after e.g. an earthquake or a fire disaster in order to analyze the degree of damage to buildings. For this application, there is no need of a high geometric accurate sensor, also SLC Camera based Oblique Camera system as the OIS-S, which uses Canon Cameras, give great results. From these data, deformation analysis to estimate the remaining building stability can be produced (Kemper 2017.)

Also these images can be accessed immediately even with a core reference. Using direct referencing methods, the nadir images data can be already overlaid in a GIS environment and build rapid access information. For the processing of the oblique images, special tools e.g. Photomesh from Skyline Soft or others on a rapid computing environment (e.g. cloud computing) are required. 


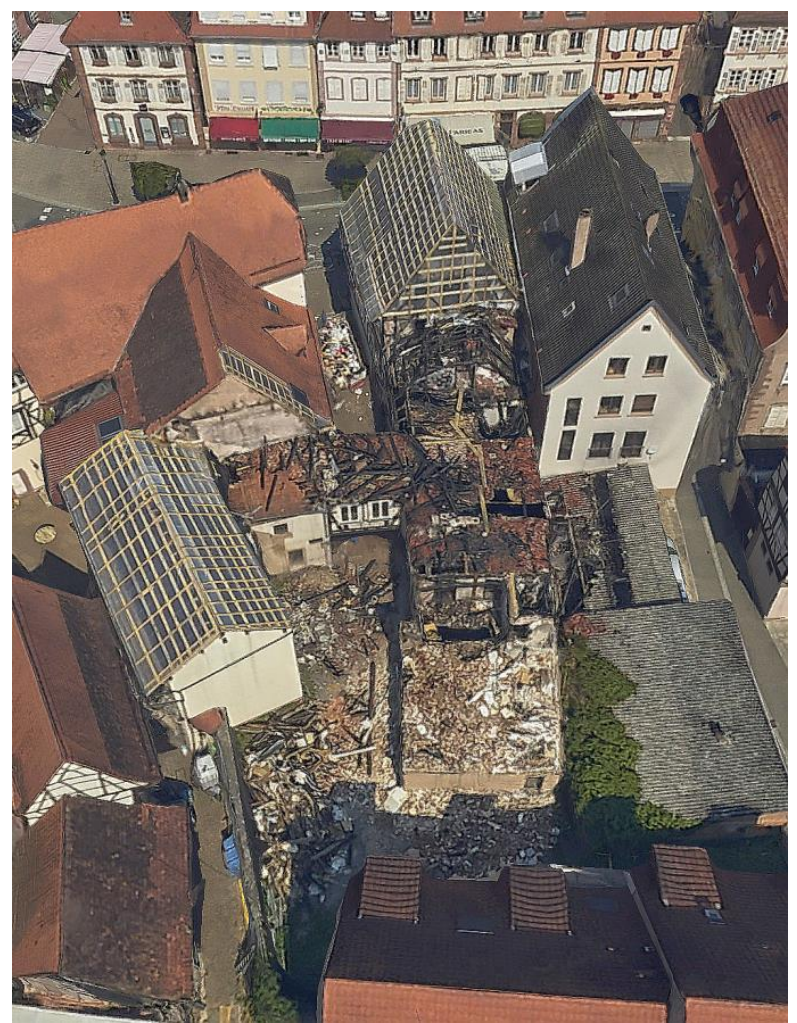

Figure 2: 3D Model showing a fire burned area of the city of Wissembourg/France generated with Skyline Software out of an OIS-X1 4B Mission 2019

\subsection{Thermal Cameras}

Thermal cameras measure the emission of longwave infrared emissions which are linked to the surface temperature. Finally, in such data, thermal anomalies are interpreted. The most common is the use of aerial thermal data for heat loss analysis and the determination of urban microclimate. It is also used for environmental monitoring of rivers and lakes/seas as well as an indication for water in the soil or urban structures (Vasel et al. 2012.). That way they can support the database with additional information about the environmental situation and the effect of urban structures.

In the monitoring process while detecting changes in the temperature, thermal cameras can be used as early warning sensors, e.g. in the warm-up phases of a geological structure (volcanoes) or environmental disasters.

Beside the application for searching people, thermal anomalies can be affected by humidity in constructions (transpiration effects), damaged power lines (e.g. defective Insulators), burning gas tubes, and many other dangerous facts. After a Disaster, Thermal images can help to identify people in the observed area. Thermal data in combination with RGB give further information on the building structure, damages, and potential water intrusion. Even small cracks in concrete can infilter water which itself reduces the thermal emission which is visible in thermal data.

Interpretation of Thermal data are complex due to variation in material emission coefficients, nevertheless context-wise can give good additional information for the crisis managers.
Better nadir the thermal camera only is an additional survey of oblique thermal information. Oblique thermal high-resolution sensor with nine heads (Aero OTS from GGS) enables highresolution nadir and oblique thermal data acquisition. That way, also facades and partly views inside the building are possible and widens the information.

A big advantage of thermal sensor ais the usability in day and nighttime. This enables the possibility to fly e.g. during the day in combination with RGB-NIR or hyperspectral and during nighttime in combination with Lidar.

Due to the small data volume of thermal images they can be accessed immediately after the flight or already downlinked using modern mobile networks (5G) to a control centre. Using direct referencing methods, the thermal data can be part of the rapid access to GIS.

\subsection{Lidar}

Lidar can generate dense DSM and also DTM using last Pulse information and algorithms to remove artificial objects and vegetation. The height information is received by direct distance measurements and results in an accurate and very dense point cloud. This represents the surface perfectly and builds an excellent data-source for the preparedness plan.

Dual-frequency Lidar scanners can measure the depth of water and can be used for bathymetry. This gives important information on changes in the shoreline e.g. after a Tsunami.

Lidar supports the disaster management by analyzing changes in the DSM before and after an "event" (Steinle and Bähr 1999.). The advantage of Lidar is that besides rain and clouds, no other weather conditions limit their use. This enables aerial missions at nighttime e.g. in combination with Thermal cameras.

After a mission, the data requires a certain level of preprocessing. Access can be realized roughly about one day after the flight.

Innovations are in the development of Lidar systems using RGB laser-beams that enable the generation of colored point clouds that represent height and surface analysis. That way it becomes an image sensor that enables to capture also visible data in the night time. Even not being equal to an aerial image, it widens the surveying time-frame for capturing data dramatically. Small and powerful Lidarsystems can be carried by UAS systems (e.g. AeroSpector) and capture missions of 40 flight time using lidar and high-resolution aerial imaging sensors in combination.

\subsection{Corona Camera}

The GGS Corona Camera is designed for a high frequent RGB Camera beside a daylight filtered UV amplified sensor. It helps to monitor discharge effects on electric powerlines and electric systems to indicate issues that can lead to complete shutdowns or even in the worst case to danger to life or fire hazards. 
The combination of these 2 sensors enable the direct overlay of the discharge spikes over an RGB Image

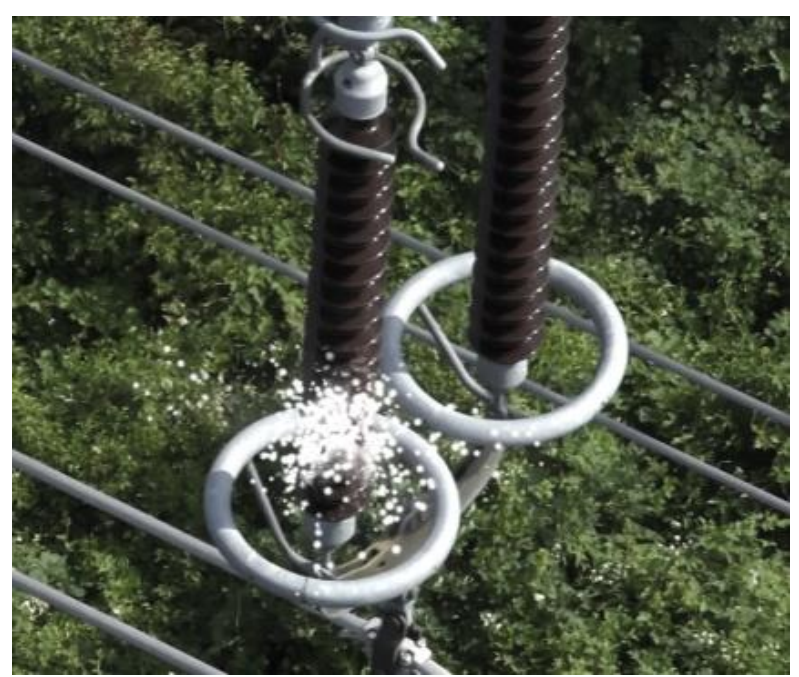

Figure 3: Discharge effects on a powerline (GGS GmbH 2019.)

\section{PLATFORMS}

As described in the previous part, some platforms are closely linked to the sensor. Satellites travel on discrete orbits and adaptations of the observed area are possible only by rotating the angle of view of the sensor. With the exception of Radar, satellites are depended also on weather conditions. But they are always operational and are able to collect data meanwhile overflying the project area. The resolutions are predefined.

Aircraft are far more flexible for carrying various sensors, adopt the resolution by the variation of flight altitude and sensor, and are flexible for flight time. Nevertheless, they need a certain infrastructure and have to be available together with equipment and crew for an aerial survey. Some sensors have been developed for generating ultra-high resolution on Helicopters. This enables closer distance to the objects, lower airspeed, and short take-off and landing area. However, the costs are far higher than for a wing based aircraft.

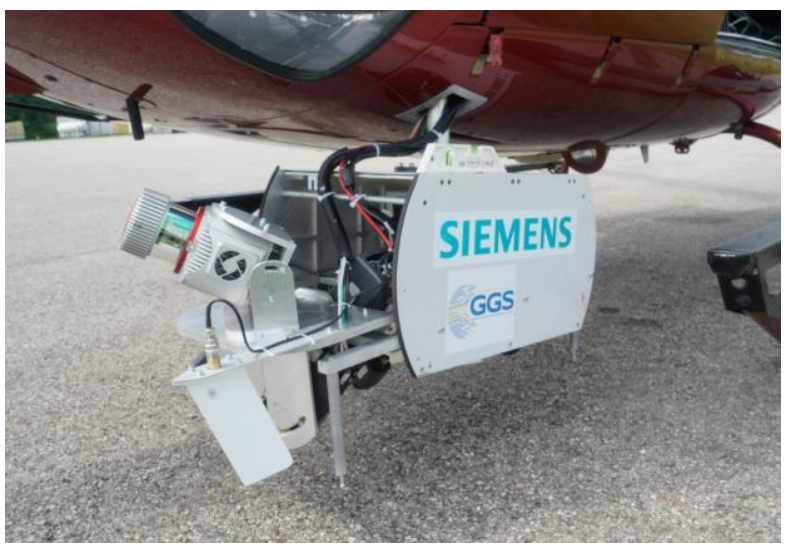

Figure 4: Monitoring system with 14 sensors for powerline monitoring, adaptable also for disaster mapping (Kemper 2018.)
Nevertheless sensors became smaller, lighter and better and also small aircraft as Trikes, Gyrocopters and UAS can be used.

Innovative platforms are UAVs for monitoring and mapping small areas. The advantages of such UAVs are that they can fly inside the urban canyons and might monitor the facades very detailed. Some of them are capable to fly inside a building and can assist in searching people. These UAVs can be transported to the project areas easily but you must have certain access to these areas.

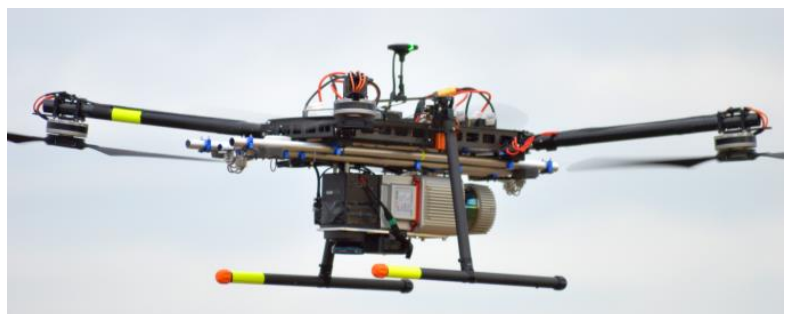

Figure 5: AeroSpector carrying a Riegl VUX Sys Lidar and a PhaseOne iXM-RS 150 Camera for more than 40' (Business Geomatics 2019.)

UAVs have a short endurance and are limited by their payload. But they can operate in areas which are not accessible by aircraft or satellites and work that way complementary.

UAVs will surely be more and more part of the disaster management on a detailed level. Today equipped with video live cams using RGB and Thermal IR, they assist in looking inside buildings and behind. Thus, they can continue with the aerial survey where airborne anomalies have been detected.

\section{AI}

$\mathrm{AI}$ is a striving and actual topic in many scientific fields and methods or concepts of machine learning are applied vividly in research. Concerning the topic of DRM an enhanced analysis of Risk or Post-Event Damages is possible. Further methods from AI were recognized as beneficial for GIS (Egenhofer and Frank 1990.). As the concept of AI in GIS was proposed in the 1990s several concepts of Machine Learning have found their way into Geospatial Analysis (Domshlak et al. 2011.). Machine Learning is often given as the "science of computer modelling of learning processes", which means that it empowers a machine to use given information and theory for the improvement of the machine's knowledge (Xueqiao Huang and John R. Jensen 1997.)

Machine Learning as one of the powerful tools from AI in GIS is discussed in detail by several scientific researchers (Xueqiao Huang and John R. Jensen 1997; Allen et al. 2016; Mojaddadi et al. 2017; Chen et al. 2018.) where it is used for geospatial risk assessments and susceptibility maps. Looking at the advanced machine learning application in Chang et al. 2019. several techniques like Random Forest, Logistic Regression and Support Vector Machine find their way into Geospatial Analysis for a Landslides Susceptibility Mapping in Taiwan. Results reveal, that several of the introduced and conducted techniques can offer a valuable add on to Susceptibility Mapping and Disaster Risk Management in general. 
Nowadays research uses these approaches to train computers by giving them a training data set and teach them several rules or indicators, which give it a certain directive. Machine Learning is now able to filter out noise from Twitter data to reduce the amount of information to the relevant one for a Disaster Scenario (Allen et al. 2016.). Further, Machine Learning is useful for the Improvement of the Disaster Communication, as it enhances the appropriation of Response mechanisms and the awareness of local stakeholders (Ighodaro Ogie et al. 2018.). First International Organizations do recognize the high potential of Machine Learning for their Disaster Risk Management Programs, like the World Bank (GFDRR 2018.) Artificial Intelligence plays a crucial role in future Risk analysis and communication with local Managers.

\section{CONCLUSION}

Today we have a good variation of sensors, software, and carriers to support Disaster Risk Management.

Big technological developments opened up abundant possibilities to support Disaster Risk Management processes capturing, processing, and representing geospatial data in all phases of a disastrous occurrence. Taking into consideration the entrepreneurship working on hardware and platform system e.g. UAV systems and thermal, RGB or oblique sensors, faster data capturing and availability is created, due to smaller and more powerful sensors. Thermal cameras and UV cameras, and in general the sensor combination gathers information together, which is essential for disaster response. Combining these new improvements with the several AI methods, i.e. Machine Learning and GIS software applications a very promising path towards integrated and interdisciplinary Disaster Risk Management. We never have been so flexible in the way of capturing remotely sensed data besides that the data quality improved dramatically. The idea to join different sensors generates new strategies in analyzing the results. There is a big task in the data-fusion and Meta-data management to be done beforehand. We should make use of the technology to support our society in times of crisis and make a pathway for sustainable development and resilient communities.

\section{REFERENCES}

Abbas S.H., Srivastava R.K., Tiwari R.P., Bala Ramudu P. 2009.GIS-based disaster management: A case study for Allahabad Sadar sub-district (India). Management of Environmental Quality: An International Journal 20 (1), 33-51.

Abdel-Aziz Belal, Hassan R. El-Ramady, Elsayed S. Mohamed, Ahmed M. Saleh 2014.Drought risk assessment using remote sensing and GIS techniques. Arabian Journal of Geosciences 7 (1), 35-53. https://link.springer.com/content/pdf/10.1007/s12517-012-0707-2.pdf.
Agrawal, N. (Ed.) 2018.Natural Disasters and Risk Management in Canada. An Introduction. Springer Netherlands, Dordrecht.

Allen, C., Tsou, M.-H., Aslam, A., Nagel, A., Gawron, J.-M. 2016.Applying GIS and Machine Learning Methods to Twitter Data for Multiscale Surveillance of Influenza. PloS one 11 (7), e0157734.

Alpar, B., Gazioglu, C., Altinok, Y., Yücel, Z.Y., Dengiz, S. 2004.Tsunami Hazard Assessment in Istanbul using by high resolution satellite data (IKONOS) and DTM. ISPRS - International Archives of the Photogrammetry, Remote Sensing and Spatial Information Sciences 20,

Altan, M.O., Kemper, G. 2008.Sytem Architecture for Earthquake, Tsunami Preapredness and Warning. ISPRS International Archives of the Photogrammetry, Remote Sensing and Spatial Information Sciences 37 (B4), 10611072.

Altan, M.O., Kemper, G. 2016.Innovativ Airborne Sensors for Disaster Management. ISPRS - International Archives of the Photogrammetry, Remote Sensing and Spatial Information Sciences 41 (B8), 11-16. 10.5194/isprsarchives-XLIB8-11-2016.

Bala, P., Santhi, T., Shinde, R. 2017.GIS and Remote Sensing in Disaster Management. Imperial Journal of Interdisciplinary Research (IJIR) 3 (5), 732-737.

Bouwer, L.M. 2011.Have Disaster Losses Increased Due to Anthropogenic Climate Change? Bulletin of the American Meteorological Society 92 (1), 39-46.

Business Geomatics 2019.Bis zu 25 Kilogramm Startgewicht: GGS stellt neuen Quadrocopter AeroSpector auf der Intergeo vor. Business Geomatics. http://www.business-geomatics.com/bgx/2019/09/09/bis-zu-25-kilogramm-startgewicht-ggs-stellt-neuen-quadrocopter-aerospector-auf-derintergeo-vor/.

Canevari-Luzardo, L., Bastide, J., Choutet, I., Liverman, D. 2017.Using partial participatory GIS in vulnerability and disaster risk reduction in Grenada. Climate and Development 9 (2), 95-109.

Chang, K.-T., Merghadi, A., Yunus, A.P., Pham, B.T., Dou, J. 2019.Evaluating scale effects of topographic variables in landslide susceptibility models using GIS-based machine learning techniques. Scientific reports 9 (1), 12296.

Chen, W., Peng, J., Hong, H., Shahabi, H., Pradhan, B., Liu, J., Zhu, A., Pei, X., Duan, Z. 2018.Landslide susceptibility modelling using GIS-based machine learning techniques for Chongren County, Jiangxi Province,

China. Science of The Total Environment 626, 1121-1135. 
Domshlak, C., Hüllermeier, E., Kaci, S., Prade, H. 2011.Preferences in AI: An overview. Artificial Intelligence $175(7-8), 1037-1052$

Egenhofer, M., Frank, A. 1990.LOBSTER: Combining AI and database techniques for GIS. Photogrammetric Engineering \& Remote Sensing 56 (6), 919-926.

FAO, Chapter 6 - The Functioning of a GIS. http://www.fao.org/3/w0615e/W0615E06.htm.

Forslund, L. 2016.Development of methods for flood analysis and response in a Web-GIS for disaster management. Student thesis series INES. http://lup.lub.lu.se/student-papers/record/8883940/file/8883946.pdf.

GFDRR 2018.Machine Learning for Disaster Risk Management. https://understandrisk.org/publication/machine-learning-for-disaster-risk-management/.

GGS GmbH 2019.Multi Sensor Integration of Phase One Industrial Cameras for Power Line Monitoring by GGS. https://www.youtube.com/watch?v=ogZ0GDi6_VA\&feature=youtu.be .

Haigh, R. 2017.Disaster Management Lifecycle. Learning Package.

Ighodaro Ogie, R., Castilla Rho, J., Clarke, R. 2018.Artificial Intelligence in Disaster Risk Communication: A Systematic Literature Review - IEEE Conference Publication. 10.1109/ICT-DM.2018.8636380. (Accessed 13 March, 2020).

Kemper, G. 2017.Hochauflösende Multisensor-Systeme. In: Kersten, T.P. (Ed.), Kulturelles Erbe Erfassen und bewahren. Von der Dokumentation zum virtuellen Rundgang, Deutsche Gesellschaft für Photogrammetrie, Fernerkundung und Geoinformation, p. 170.

Kemper, G. 2018.Multi Sensor Setup for various applications. Photogrammetric Society Hungary,

Khan, N. 2013.Flood Prediction and Disaster Risk Analysis using GIS based wireless sensor Networks. A Review. Journal of Basic and Applied Scientific Research 3 (8), 632-643.

Kienberger, S., Steinbruch, F. 2005.P-GIS and disaster risk management: Assessing vulnerability with P-GIS methods. Experiences from Búzi, Mozambique.

Laituri, M., Kodrich, K. 2008.On Line Disaster Response Community: People as Sensors of High Magnitude Disasters Using Internet GIS. Sensors (Basel, Switzerland) 8 (5), 3037-3055.

Maskrey, A. 2011.Revisiting community-based disaster risk management. Environmental Hazards 10 (1), 42-52.
Mojaddadi, H., Pradhan, B., Nampak, H., Ahmad, N., Ghazali, A.H.b. 2017.Ensemble machine-learning-based geospatial approach for flood risk assessment using multi-sensor remote-sensing data and GIS. Geomatics, Natural Hazards and Risk 8 (2), 1080-1102.

Niculescu, S., Lardeux, C., Güttler, F., Rudant, J.-P. 2010.Multisensor Systems and Flood Risk Management. Application to the Danube Delta Using Radar and Hyperspectral Imagery. Télédéteccion 9 (3-4), 271-288.

O`Brien, G., O`Keefe, Rose, Joanne, Wisner, B. 2006.Climate change and disaster management. Disasters 30 (1), 6480 .

Steinle, E., Bähr, H.-P. 1999.Laserscanning for Change Detection. In: Altan, M.O., Gründig, L. (Eds.), Third TurkishGerman joint geodetic days: Towards a digital age. Istanbul Technical University.

Tronin, A. 2010.Satellite Remote Sensing in Seismology. A Review. Remote Sensing 2 (1), 124-150.

United Nations Office for Disaster Risk Reduction 2015.Sendai Framework for Disaster Risk Reduction 2015 2030.

van Aalst, M.K. 2006. The impacts of climate change on the risk of natural disasters. Disasters 30 (1), 5-18.

Vasel, R., Kemper, G., Schuhmann, R., Königer, F. 2012.New Airborne Thermal Infrared Photogrammetric Applications and Sensors for Moisture Detection. ISPRS - International Archives of the Photogrammetry, Remote Sensing and Spatial Information Sciences,

Xueqiao Huang and John R. Jensen 1997.A Machine-Learning Approach to Automated Knowledge-Base Building for Remote Sensing Image Analysis with GIS Data. Photogrammetric Engineering \& Remote Sensing 63 (10), 1185-1194. 